\title{
Predicting the stability and electronic structure of alkali metal aurides
}

\author{
Axel M. Gaona Carranza, ${ }^{*}$ Jesús M. Siqueiros, and Jonathan Guerrero Sánchez ${ }^{\dagger}$ \\ Centro de Nanociencias y Nanotecnología,Universidad Nacional Autónoma de México, \\ Km. 107, Apdo. 14 Carretera Tijuana-Ensenada, Baja California, México \\ Reyes García Díaz \\ CONACyT-Facultad de Ciencias Fúsico Matemáticas, Universidad Autónoma de Coahuila, \\ Unidad Camporedondo, Edif. A, 25000, Saltillo, Coahuila, México
}

(Dated: July 17, 2021)

\begin{abstract}
Density functional theory calculations of phonon modes predict that some compounds of the alkali metal aurides family, general formula $\mathrm{A}_{2} \mathrm{MAu}_{6}\left(\mathrm{~A}=\mathrm{K}^{+}, \mathrm{Rb}^{+}\right.$or $\mathrm{Cs}^{+} ; \mathrm{M}=\mathrm{Ti}, \mathrm{Zr}, \mathrm{Hf}, \mathrm{Sn}$ or $\left.\mathrm{Pb}\right)$, have stable three-dimensional phase with a double perovskite-type structure and cubic $F m \overline{3} m$ space group $\left(\mathrm{K}_{2} \mathrm{PtCl}_{6}\right.$-type). Bader's charge analysis shows that most electron density is located within the six atoms at the octahedra vertices like double perovskite halides. However, the short spacing between $\mathrm{Au}$ anions enables d-orbital interactions between them. Compounds of this family, with group 4 metals only, carry conduction states around the $\Gamma$ point $(\mathrm{k}=0)$. On the other hand, compounds with group 14 metals possess more conduction states around all the Brillouin zone and have electron pockets in their bandstructure. These compounds provide further insights into the unusual anionic behavior of gold and present other alternatives for the construction of divergent nanodevices.
\end{abstract}

\section{INTRODUCTION}

Transition metals with anionic character are present in several compounds such as $\mathrm{AuCs}^{[1-3]}, \mathrm{AuRb}^{[4]}$ with $\mathrm{CsCl}$ structure ${ }^{[5-7]}, \mathrm{Cs}_{3} \mathrm{AuO}^{[8]}$, and $\mathrm{Rb}_{3} \mathrm{AuO}^{[9]}$ ternary oxides. Also in the PtBa anisotropic conductor ${ }^{[10]}$, along with other barium platinides ${ }^{[11]}$. These compounds have in common the coexistence of low electronegative alkali and alkaline-earth metal elements ( $\mathrm{Cs}, \mathrm{Rb}$, or $\mathrm{Ba}$ ) with heavy transition metals ( $\mathrm{Pt}$ or $\mathrm{Au}$ ), which have high electron affinities: 2.12 and $2.30 \mathrm{eV}$ respectively ${ }^{[12]}$. The $\mathrm{Au}^{-}$ anion is present even in compounds with polar molecules such as caesium auride ammonia $(1 / 1), \mathrm{CsAu} \cdot \mathrm{NH}_{3}{ }^{[13]}$, and organic molecules such as tetramethylammonium auride $\mathrm{C}_{4} \mathrm{H}_{12} \mathrm{NAu}^{[14]}$.

The unique properties of gold and platinum as anions are due to their position in the periodic table, in which special relativity effects strongly influence their properties $^{[15,16]}$. The most important effects in gold are the large radial contractions of the $6 \mathrm{~s}$ shell as well as $5 \mathrm{~d}$ shell expansions ${ }^{[17-20]}$.

Individual molecules of group 4 metal tetra-aurides, formula $\mathrm{MAu}_{4}(\mathrm{M}=\mathrm{Ti}, \mathrm{Zr}, \mathrm{Hf})$, have been theoretically predicted. In such species, gold has a formal charge of $(-1)$ and acts as a ligand of the metallic center, analogous to the halogen series ${ }^{[21]}$. Also, photoelectron spectroscopy measurements combined with density functional theory (DFT) studies confirm the existence of neutral and anionic titanium tetra-auride clusters $\left(\mathrm{TiAu}_{4}\right.$ and $\left.\mathrm{TiAu}_{4}{ }^{-}\right)$with metastable tetrahedral $\left(\mathrm{T}_{\mathrm{d}}\right)$ and stable but slightly distorted tetrahedral $\left(\mathrm{D}_{2 \mathrm{~d}}\right)$ structures, respec-

\footnotetext{
* g6_gaon17@ens.cnyn.unam.mx

† guerrero@cnyn.unam.mx
}

tively. These Au-Ti clusters have multiple bond characters due to the Au $5 \mathrm{~d}$ and Ti $3 \mathrm{~d}$ back donation ${ }^{[22]}$.

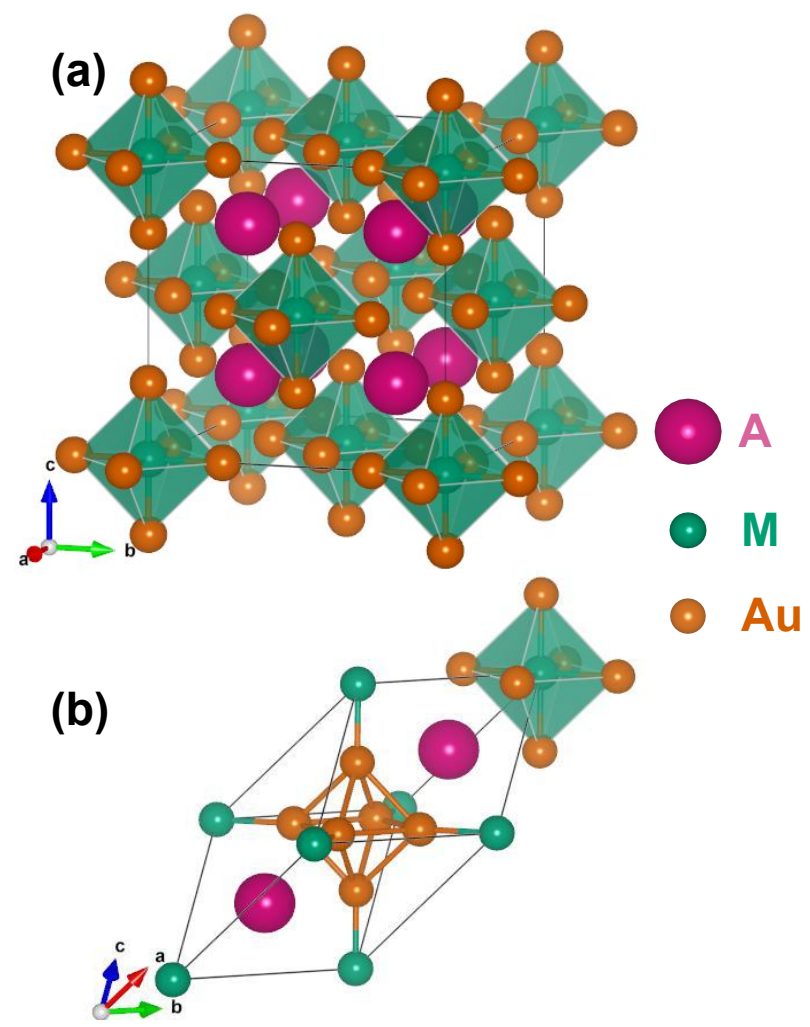

FIG. 1. Schematic representations of (a) face centered cubic unit cell and (b) primitive cell of the crystal structure of the $\mathrm{A}_{2} \mathrm{MAu}_{6}$ family. Colorblind-friendly Hex code colors: $\mathrm{A}=$ Hopbush (\#cc79a7); $\mathrm{M}=$ Dark cyan (\#009e73); $\mathrm{Au}=$ Tenne (\#d55e00). 
On the other hand, in response to the necessity of new stable and efficient photovoltaic materials, recent investigations seek to predict novel compounds analogous to hybrid halide perovskites ${ }^{[23]}$. Among these predictions, the existence of a family of inorganic and hybrid double perovskites with methylammonium or formamidinium $\left(\mathrm{MA}^{+}\right.$or $\mathrm{FA}^{+}$) based on Ti has been demonstrated theoretically and experimentally. This family has general formula $\mathrm{A}_{2} \mathrm{TiX}_{6}\left(\mathrm{~A}=\mathrm{K}^{+}, \mathrm{Rb}^{+}, \mathrm{Cs}^{+}, \mathrm{In}^{+}, \mathrm{MA}^{+}\right.$ or $\mathrm{FA}^{+} ; \mathrm{X}=\mathrm{Cl}, \mathrm{Br}$, or I) ${ }^{[24]}$.

In this computational-theoretical study we predict a promising new family of alkali metal aurides with a double perovskite structure, cubic space group $F m \overline{3} m$ $\left(\mathrm{K}_{2} \mathrm{PtCl}_{6}\right.$-type $)$ and general formula $\mathrm{A}_{2} \mathrm{MAu}_{6}\left(\mathrm{~A}=\mathrm{K}^{+}\right.$, $\mathrm{Rb}^{+}$or $\mathrm{Cs}^{+} ; \mathrm{M}=\mathrm{Ti}, \mathrm{Zr}, \mathrm{Hf}, \mathrm{Sn}$ or $\mathrm{Pb}$ ). The structure is composed of a solid-state framework of $\left[\mathrm{MAu}_{6}\right]$ octahedra, denoted for later analysis by the capital letter "O", and $\mathrm{Au}$ suboctahedra, denoted with the lowercase letter " $O$ ", which are stabilized by the A-site cations as shown in Figure 1 (b).

Our calculations show that $\mathrm{Rb}_{2} \mathrm{TiAu}_{6}, \mathrm{Rb}_{2} \mathrm{ZrAu}_{6}$, $\mathrm{Rb}_{2} \mathrm{HfAu}_{6}, \mathrm{~K}_{2} \mathrm{TiAu}_{6}, \mathrm{~K}_{2} \mathrm{ZrAu}_{6}, \mathrm{~K}_{2} \mathrm{HfAu}_{6}, \mathrm{Cs}_{2} \mathrm{PbAu}_{6}$ and $\mathrm{Cs}_{2} \mathrm{SnAu}_{6}$ compounds are dynamically stable. We divided them into two subfamilies. While the electronic and phonon structures of the six compounds having group 4 metals are similar, they differ from the other two possessing group 14 metals. These differences are related to the interaction of $d$ - or $p$-orbitals from the tetravalent metal ion (M) with the six surrounding gold anions in the octahedral geometry.

The phonon structures display a variety of maximum frequencies standing from 3.5 to $7 \mathrm{THz}$, which depend mostly on the atomic mass of the $\mathrm{M}$ metal and are much lower than those of most materials. They also exhibit a series of acoustical-optical and optical-optical phonon gaps that can be modulated by making specific ionic substitutions. This family of materials offer a case study of phonon structure engineering by increasing or reducing the phonon gap(s) through the substitution of the alkali or tetravalent metal ions.

\section{METHODOLOGY}

First-principles computations based on the KohnSham ${ }^{[25]}$ density functional theory (DFT) were carried out using the VASP ${ }^{[26]}$ package. For the exchangecorrelation potential, the General Gradient Approximation (GGA) functional given by Perdew, Burke and Ernzerhof $(\mathrm{PBE})^{[27]}$ was employed. We used projectoraugmented-wave $(\mathrm{PAW})^{[28]}$ pseudopotentials whose valence electron configurations are shown in Table I of the Supplemental Information (SI). Since we deal with heavy elements, the spin-orbit coupling (SOC) effect was employed through a second-variation method as implemented in the VASP code ${ }^{[29]}$. A plane-wave basis set is applied with a kinetic-energy cutoff of $600 \mathrm{eV}$. Longrange van der Waals interactions were included with the
Grimme (DFT-D3) method ${ }^{[30]}$. The ground state structures were determined by varying the volume isotropically. Atomic positions were relaxed until the HellmannFeynman residual forces were lower than $10^{-5} \mathrm{eV} / \AA$ per atom. In order to achieve convergence of the electronic self-consistent cycle, we set a total energy difference of $10^{-8} \mathrm{eV}$. Monkhorst-Pack ${ }^{[31]} \Gamma$-centered integration grids of $8 \times 8 \times 8$ were used to sample the first Brillouin Zone during the lattice parameter and atomic position optimizations. Denser meshes of $24 \times 24 \times 24$ were used to calculate the electronic properties. PyProcar code was used for the electronic bandstructure post-processing ${ }^{[32]}$. The $\mathrm{k}$-path used in the reciprocal space for band structure calculations, shown in Fig. 2, was determined by AFLOWonline tools ${ }^{[33]}$. In order to find the electronic density distribution, a topological analysis based on the atomsin-molecules (AIM) theory of Bader ${ }^{[34]}$ has been made, as implemented by Henkelman's group code ${ }^{[35-38]}$. Bonding and antibonding energy regions for different chemical species interactions were determined by the projected Crystal Orbital Hamilton Population (pCOHP), as implemented in LOBSTER ${ }^{[39-41]}$. The PHONOPY code $^{[42]}$ was used to perform lattice-dynamics calculations using the finite-displacements approach from interatomic force constants obtained by VASP Hellman-Feynman forces and for the phonons post-processing. The phonon calculations were performed for $2 \times 2 \times 2$ supercells.

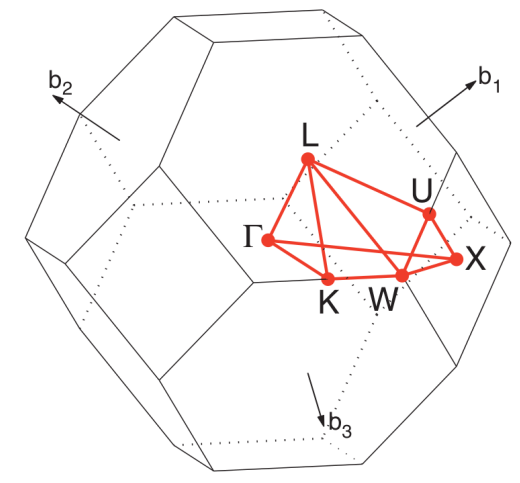

FIG. 2. Brillouin zone of FCC lattice. Path: Г-X$\mathrm{W}-\mathrm{K}-\mathrm{\Gamma}-\mathrm{L}-\mathrm{U}-\mathrm{W}-\mathrm{L}-\mathrm{K} \mid \mathrm{U}-\mathrm{X}$. Obtained from Creative Commons $\mathrm{AFLOW}^{[33]}$.

\section{RESULTS AND DISCUSSION}

Our target is to analyze the dynamical stability, electronic properties, and the effect of the spin-orbit coupling in the electronic structure of the family of alkali metal aurides. The interplay between chemical species was examined by projecting the vibrational and electronic densities of states, as well as determining the electronic density distribution through Bader's AIM theory, which provides a rigorous quantum-mechanical foundation to key concepts of the chemical structure such as ionicity, coordination index, or oxidation states based on topological analysis 
of the density distribution ${ }^{[43]}$. Additionally, the pCOHP analysis was used to interpret the bonding interactions.

First, we made an isotropic optimization for the crystal structure of all the possible combinations of the $\mathrm{A}_{2} \mathrm{MAu}_{6}$ auride family. In order to have overall charge neutrality on the system, we made ionic substitutions of every group 1 alkali ion (A), and group 4 or group 14 tetravalent metal $(\mathrm{M})$ in the primitive cell shown in Fig. 1 (b). The lattice parameters, $\mathrm{M}-\mathrm{Au}, \mathrm{A}-\mathrm{Au}, \mathrm{Au}-\mathrm{Au}$ octahedral $(O), \mathrm{Au}$ - $\mathrm{Au}$ suboctahedral $(o)$ interatomic distances and phonon dynamical stability "check" of all these compounds can be found in Table II of the SI. The eight dynamically stable compounds are shown in Table I.

TABLE I. Optimized distances for the dynamically stable compounds (in $\AA$ ). " $a$ " stands for lattice parameter, $(O)$ and (o) for $\mathrm{Au}-\mathrm{Au}$ octahedral and $\mathrm{Au}-\mathrm{Au}$ suboctahedral interatomic distances respectively.

\begin{tabular}{lccccc}
\hline \hline & $a$ & $\mathrm{M}-\mathrm{Au}$ & $\mathrm{A}-\mathrm{Au}$ & $O$ & $o$ \\
\hline $\mathrm{Rb}_{2} \mathrm{TiAu}_{6}$ & 6.735 & 2.622 & 3.376 & 3.709 & 3.025 \\
$\mathrm{Rb}_{2} \mathrm{ZrAu}_{6}$ & 6.865 & 2.721 & 3.445 & 3.849 & 3.015 \\
$\mathrm{Rb}_{2} \mathrm{HfAu}_{6}$ & 6.839 & 2.698 & 3.430 & 3.816 & 3.022 \\
\hline $\mathrm{K}_{2} \mathrm{TiAu}_{6}$ & 6.626 & 2.592 & 3.322 & 3.665 & 2.961 \\
$\mathrm{~K}_{2} \mathrm{ZrAu}_{6}$ & 6.771 & 2.694 & 3.398 & 3.881 & 2.959 \\
$\mathrm{~K}_{2} \mathrm{HfAu}_{6}$ & 6.746 & 2.673 & 3.385 & 3.780 & 2.965 \\
\hline $\mathrm{Cs}_{2} \mathrm{SnAu}_{6}$ & 6.902 & 2.785 & 3.468 & 3.938 & 2.963 \\
$\mathrm{Cs}_{2} \mathrm{PbAu}_{6}$ & 6.991 & 2.873 & 3.518 & 4.064 & 2.926 \\
\hline \hline
\end{tabular}

From now on, we focus on the study of some of the representative members of the $\mathrm{A}_{2} \mathrm{MAu}_{6}$ family: $\mathrm{Rb}_{2} \mathrm{TiAu}_{6}$, $\mathrm{Cs}_{2} \mathrm{SnAu}_{6}$ and $\mathrm{Cs}_{2} \mathrm{PbAu}_{6}$. First, we discuss the lattice vibrations from the phonon band structures and vibrational density of states (VDOS) in Section A. Then, we demonstrate the anionic character of gold and bonding interactions through Bader's AIM theory and COHP analyses in Section B. Finally, we analyze the electronic band structures and their effects by SOC interactions in section C.

\section{A. VIBRATIONAL PROPERTIES}

The phonon dispersion plot shown in Fig. 3 (a) indicate that the $\mathrm{Rb}_{2} \mathrm{TiAu}_{6}$ compound is dynamically stable due to all its acoustical and optical modes have positive frequencies. Imaginary frequencies $(\omega<0)$ are usually interpreted as structural instabilities or phase transitions. The VDOS, shown in Fig. 3 (b), reveals that from low frequencies up to $2 \mathrm{THz}$, the acoustical branches are hybridized collective motions of all the atomic species. However, most of the vibrational modes arise from $\mathrm{Au}$ contributions. At higher frequencies, from 2 to $3 \mathrm{THz}$, the vibrational density is dominated by $\mathrm{Au}$ modes, caused by three optical phonon branches and forming a three-peak ordering present in all the VDOS belonging to the compounds from the group 4 metal auride subfamily. It is noteworthy that the top two bands forming this three-peak ordering are symmetric in the $\Gamma-X-W-K-\Gamma$ momentum path around 2.75 THz. This triple peak is followed by a pronounced crest caused primarily by A ions, $\mathrm{Rb}$ in this case, around 3.5 THz. Subsequently, there is an optical-optical phonon gap (OOPG) of $1.68 \mathrm{THz}$, at which atomic vibrational modes are forbidden. The value of this frequency gap is the highest of the auride family studied here, as can be seen in Fig. 4, and is caused primarily by the considerable difference between the small atomic mass of $\mathrm{Ti}$ and the bigger ones of $\mathrm{Rb}$ and $\mathrm{Au}$. Finally, reaching the top frequencies, from 5.3 up to $6.8 \mathrm{THz}$, the optical modes are caused by three high-frequency phonon branches driven by $\mathrm{Ti}$ atoms with minor contributions from $\mathrm{Au}$.

The total VDOS from the six compounds having group 4 metals are shown in Fig. 4. The phonon dispersions and ionic contributions in the VDOS of all these compounds are shown in Figs. 1-5 of the SI. The hybridized acoustical modes at low frequencies of most compounds reach their maximum at $2 \mathrm{THz}$. However, compared with their Rb counterparts, the compounds with $\mathrm{K}$ possess higher maximal acoustical frequencies, giving rise to a hardening of the high-frequency acoustical modes and increased VDOS frequencies. This trend can be observed by comparing the K-Rb pairs in Fig. 4: the frequency values of the three-peak ordering associated with gold, the alkali-ion crest, as well as the top frequency values of the optical branches associated with $\mathrm{M}$ metal ions are higher in the presence of $\mathrm{K}$.

The top frequency values from the optical branches are strongly influenced by the atomic mass of the M metal ions. As a result of the $\mathrm{M}$ mass increase, the top frequency decreases, and the vibrations from Au ions gradually start to couple with those frequencies (See all the VDOS in the SI), leading to increases in VDOS values and narrower frequency ranges. These phenomena trigger the OOPG closing.

On the other hand, the frequency value of the alkaliion crest remains almost constant, depending on whether it is $\mathrm{Rb}$ or $\mathrm{K}$. While the crests have a slight increase in frequency in the compounds with $\mathrm{Ti}$, those with $\mathrm{Zr}$ and Hf show almost identical frequency ranges. This effect is not related to the mass of the last two chemical species. The difference between both is nearly 200\% (See Table I in SI), but with their nearly equal ion radii, which are reflected in the very similar lattice parameters, and the interatomic distances of $\mathrm{M}-\mathrm{Au}$, and $\mathrm{A}-\mathrm{Au}$ of the compounds with $\mathrm{Zr}$ and Hf, as seen in Table I. As the lattice parameter and $\mathrm{Au}-\mathrm{Au}$ octahedral $(O)$ sublattice distances increase, the available volume for the alkali-ion increases. This consequence can be verified by looking at the A-Au distances in Table I and seeing the calculated volumes by Bader's analysis in Table II, and in Tables III-IX of the SI. In other words, the presence of more extensive $\mathrm{M}$ metals increases the $\mathrm{Au}-\mathrm{Au}$ octahedral $(O)$ distances, expanding the available volume and enlarging 


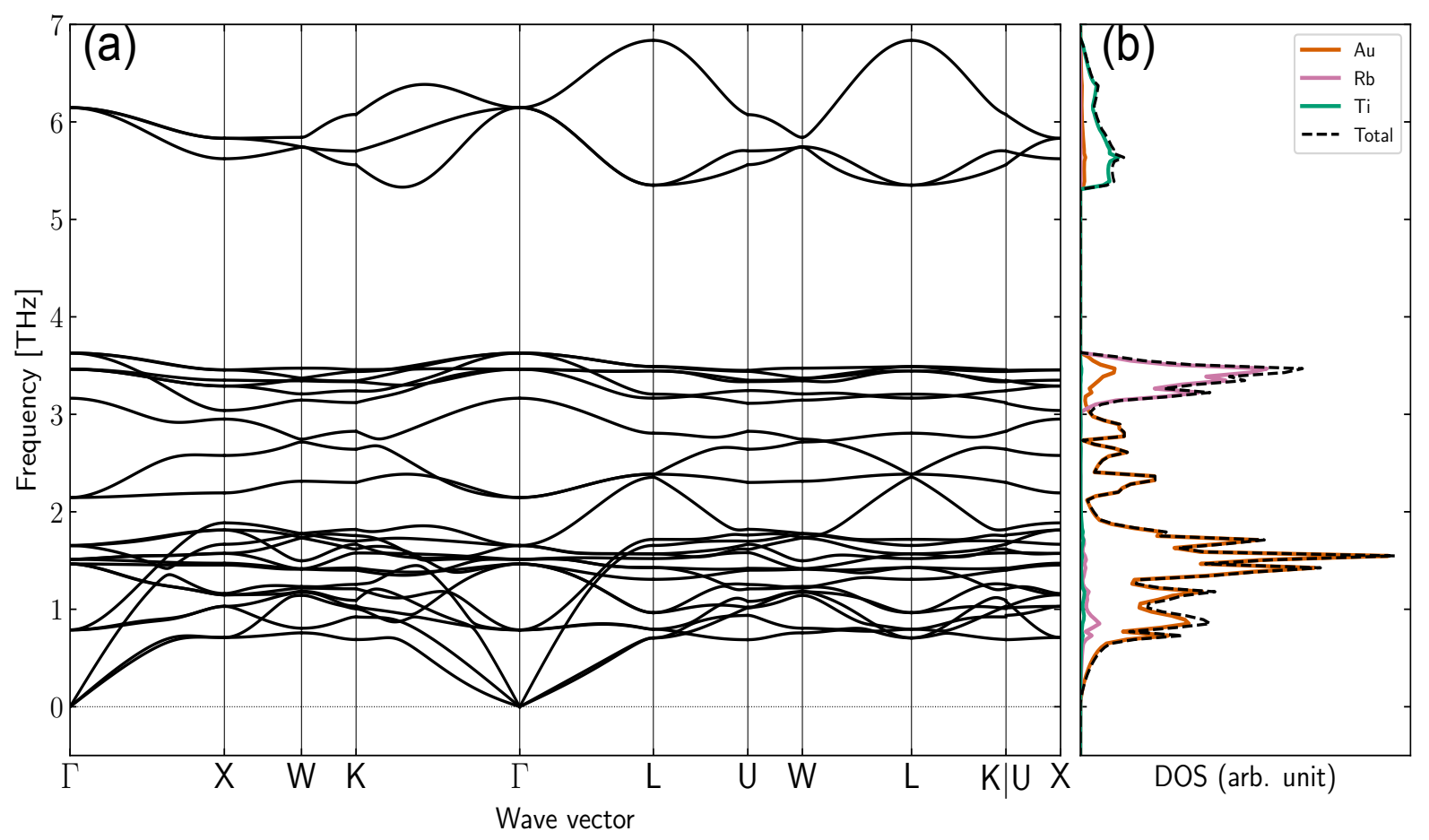

FIG. 3. Computed (a) phonon band structure of $\mathrm{Rb}_{2} \mathrm{TiAu}_{6}$ and (b) phonon density of states.

the mean-square displacements of the A alkali-ion, which decreases the frequency value of the peaks.

Finally, the frequency of the VDOS three-peak ordering from $\mathrm{Au}-\mathrm{Au}$ interactions is affected by both $\mathrm{A}$ and $\mathrm{M}$ ion masses. It was previously found that $\mathrm{K}$ ion induces a hardening in the phonon modes. On the other hand, greater $\mathrm{M}$ metal mass leads to an opening of a lowfrequency acoustical-optical phonon gap (AOPG) due to acoustical branch softenings, and slight rising of the three-peak orderings. The lack of AOPGs in compounds with $\mathrm{Ti}$ is related to a band-crossing occurring between the acoustical modes and the three-peak ordering optical phonon branches at the high symmetry L point of the Brillouin Zone between 2 and 3 THz (See Fig. 3 and Fig. 3 in SI).

The whole picture trend in Fig. 4 suggests a "V" shape traced from low- to high-frequencies by the threepeak ordering, and from high- to low-frequencies by the top optical phonon peaks. Following this "V" shape trend by specific ionic substitutions, one can induce highfrequency OOPG reductions at the same time with lowfrequency AOPG enlargements with a variety of intermediate stages. There are some special cases in the behavior of the phonon gaps, for example, in $\mathrm{K}_{2} \mathrm{TiAu}_{6}$ in that a second OOPG appears below the alkali-ion crest and is primarily caused by the mass similarity between $\mathrm{K}$ and $\mathrm{Ti}$, or in $\mathrm{K}_{2} \mathrm{ZrAu}_{6}$ and $\mathrm{K}_{2} \mathrm{HfAu}_{6}$ in which almost negligible phonon gaps are present (marked by blue diamonds in Fig. 4).

The phonon dispersions of the second subfamily mem- bers, $\mathrm{Cs}_{2} \mathrm{SnAu}_{6}$ and $\mathrm{Cs}_{2} \mathrm{PbAu}_{6}$, are shown in Fig. 5. As the previous cases, it is said that they are dynamically stable due to the lack of imaginary frequencies, and have a unique OOPG of 0.4 and $0.06 \mathrm{THz}$ respectively. The top frequency optical branches are caused by $\mathrm{M}$ metals and Au vibrational modes, around 4 and $3.4 \mathrm{THz}$ respectively.

For $\mathrm{Cs}_{2} \mathrm{SnAu}_{6}$, the compound maintains the same general phonon structure as the previously discussed compounds, starting from the acoustical branches highly dominated by gold at lower frequencies where there also exist the acoustical-optical branches crossings at L point around $2.2 \mathrm{THz}$. It also maintains the crest caused by alkali ions, in this case $\mathrm{Cs}$, at $2.9 \mathrm{THz}$, and the top frequency optical branches strongly influenced by the mass of the M metal, in this case Sn, around $4 \mathrm{THz}$.

On the other hand, the $\mathrm{Cs}_{2} \mathrm{PbAu}_{6}$ compound does not possess almost any of the previously observed vibrational properties but a more complex variety of hybridizations along the whole VDOS, as can be seen in Fig. 5 (d). This compound is the only one in the complete auride family studied here in which Au vibrational modes dominate only one top optical phonon branch. Such contrasting phenomena are attributed to the fact that $\mathrm{Pb}$ is the heavier element of the $\mathrm{A}_{2} \mathrm{MAu}_{6}$ family, and the only one with larger atomic mass than $\mathrm{Au}$ ions. As can be seen from Fig. 4, as the atomic mass from $\mathrm{M}$ ions increases, the top optical frequency value decreases. Therefore, the heavy $\mathrm{Pb}$ ion triggers an extreme frequency lowering of its optical branches, causing strong optical phonon hy- 


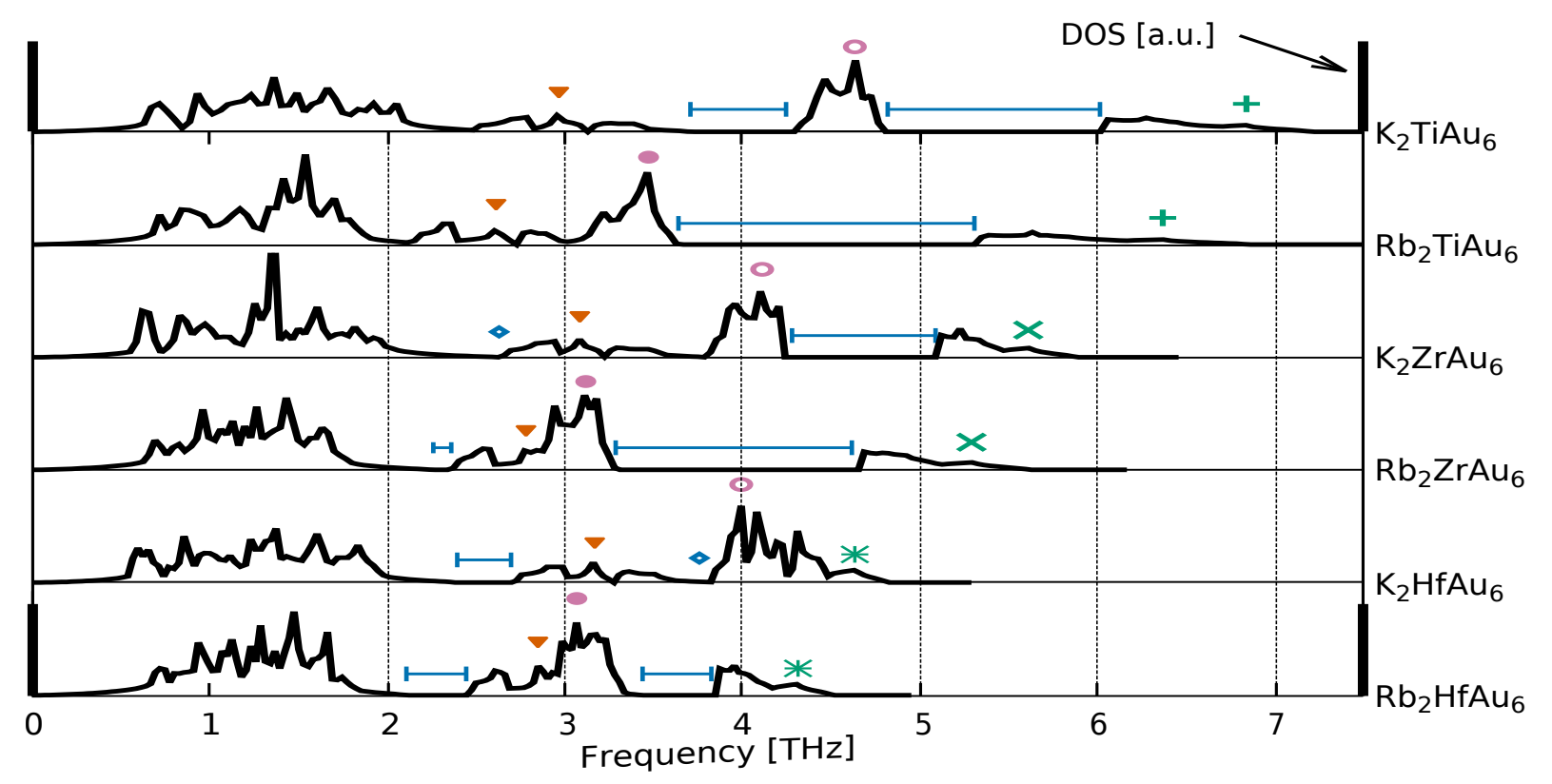

FIG. 4. Calculated VDOS for dynamically stable compounds with group 4 metals. Colorcode used from Fig. 1: Blue lines represent phonon gaps; blue diamonds $(\diamond)$ are zero-value density of states points; tenne inverted triangles $(\boldsymbol{\nabla})$ point the center peak of the Au-dominant three-peak orderings; hopbush filled $(\bullet)$ and empty (o) dots point Rb and K peaks respectively; dark cyan colored vertical crosses $(+)$, diagonal crosses $(\times)$ and asterisks $(*)$ label Ti, Zr and Hf optical peaks respectively).
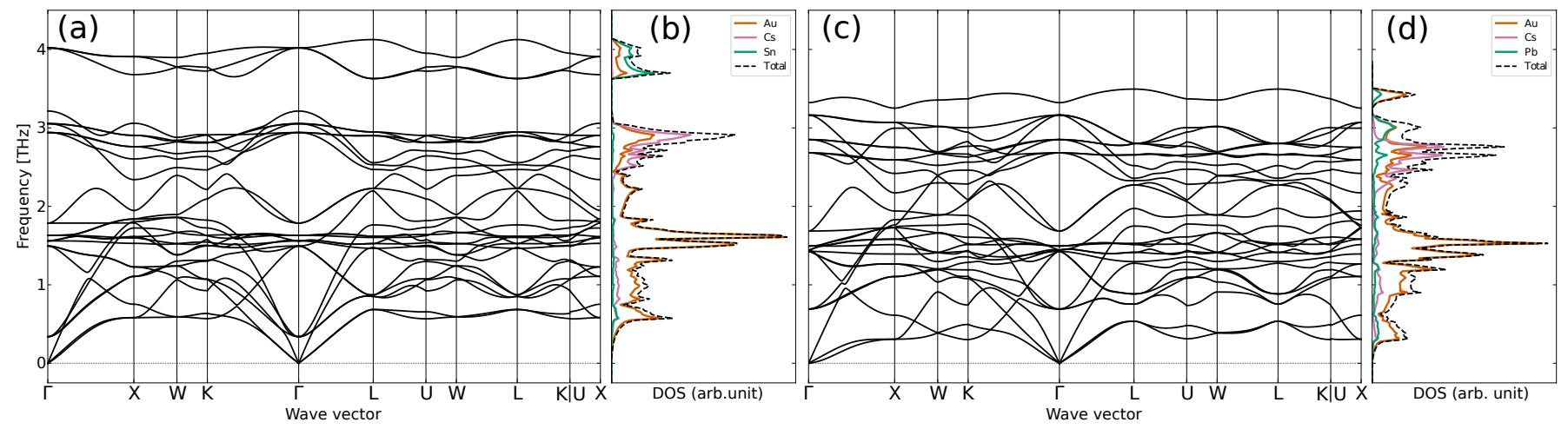

FIG. 5. Computed (a) phonon band structure and (b) phonon density of states of $\mathrm{Cs}_{2} \mathrm{SnAu}_{6}$; (c) phonon band structure and (d) phonon density of states of $\mathrm{Cs}_{2} \mathrm{PbAu}_{6}$.

bridizations and a complete phonon dispersion softening.

One reason why the $\mathrm{Cs}_{2} \mathrm{MAu}_{6}$ with group 4 metals subfamily is dynamically unstable is the big ionic radius of Cs. This is reflected as imaginary phonons at the $\Gamma$ point in phonon dispersions shown in Figs. 6-8 of the SI.

The gap variations in these materials could lead to future phonon structre engineering and phonon gap tuning ${ }^{[44]}$. For example, by engineering two materials with mismatching phonon gaps, we might have a large interfacial thermal resistance ${ }^{[45]}$.

\section{B. ELECTRONIC DENSITY DISTRIBUTION}

Atomic charges were obtained from the topological analysis of the electron density according to Bader AIM theory. The integrated valence electron densities within the atoms for $\mathrm{Rb}_{2} \mathrm{TiAu}_{6}$ are shown in Table II. In the non-relativistic case, the sum of all extra electrons in the six $\mathrm{Au}$ atoms is 2.944, while $\mathrm{Rb}$ and $\mathrm{Ti}$ atoms together lose 2.949 electrons. On the other hand, DFT-SOC functionals do not significantly change these results by obtaining 2.934 extra electrons for the six Au ions and 2.937 electrons given up by $\mathrm{Rb}$ and $\mathrm{Ti}$.

The calculated charge densities of the remaining compounds with group 4 elements are shown in Tables IIIVII of the SI. These results support the description of $\mathrm{Au}$ atoms as anionic, halogenoid species in this system. However, they do not clarify if they behave more as iodides or bromides, as this ion was observed as an intermediate between them in previous works on $\mathrm{MAu}_{4}$ tetrahedral molecules with group 4 metals $^{[21]}$ and AAu alkali auride 
TABLE II. Calculated charge per atom through Bader's AIM topological analysis for $\mathrm{Rb}_{2} \mathrm{TiAu}_{6} . e_{i}$ stands for initial valence electrons; $\rho_{B}$ and $\rho_{B-S O C}$ stands for Bader's electronic density obtained from DFT and DFT-SOC functionals, respectively. $\Omega_{\mathrm{B}}$ stands for Bader volume (in $\AA^{3}$ ).

\begin{tabular}{ccccc}
\hline \hline Atom & $e_{i}$ & $\rho_{B}$ & $\rho_{B-S O C}$ & $\Omega_{\mathrm{B}}$ \\
\hline $\mathrm{Au}(1)$ & 11 & 11.487 & 11.485 & 26.88 \\
$\mathrm{Au}(2)$ & 11 & 11.491 & 11.485 & 26.90 \\
$\mathrm{Au}(3)$ & 11 & 11.504 & 11.502 & 27.02 \\
$\mathrm{Au}(4)$ & 11 & 11.507 & 11.505 & 27.05 \\
$\mathrm{Au} \mathrm{(5)}$ & 11 & 11.481 & 11.480 & 26.82 \\
$\mathrm{Au}(6)$ & 11 & 11.474 & 11.477 & 26.76 \\
\hline $\mathrm{Rb}(1)$ & 9 & 8.268 & 8.270 & 21.64 \\
$\mathrm{Rb}(2)$ & 9 & 8.268 & 8.270 & 21.64 \\
\hline $\mathrm{Ti}(1)$ & 12 & 10.515 & 10.523 & 11.27 \\
\hline \hline
\end{tabular}

molecules ${ }^{[46]}$. As a proof of concept, Bader's charges were also calculated for $\mathrm{Rb}_{2} \mathrm{TiI}_{6}$ and $\mathrm{Rb}_{2} \mathrm{TiBr}_{6}$ structures with optimized lattice parameters of $8.108 \AA$ and $7.509 \AA$ respectively. The calculated electron densities for both in the non-relativistic cases are shown in Tables VIII and IX.

The anionic character of $\mathrm{Au}$ in $\mathrm{Rb}_{2} \mathrm{TiAu}_{6}$ is similar to the halogens in $\mathrm{Rb}_{2} \mathrm{TiI}_{6}$. In $\mathrm{Rb}_{2} \mathrm{TiI}_{6}$ and $\mathrm{Rb}_{2} \mathrm{TiBr}_{6}$, the halogens gain 3.291 and 3.522 electrons, respectively, while the donor species ( $\mathrm{Rb}$ and $\mathrm{Ti}$ ) confer 3.296 and 3.525 electrons. This differs from the I-Br intermediate picture of Au from Refs. [21] and [46], which is attributed to the fact that these systems do not have a 1:1 relation between the $\mathrm{A}$ and Au chemical species, but a 1:3 relation. Another reason is that here the compound is studied as a solid-state framework combination between both $\mathrm{AAu}$ and group- $4 \mathrm{MAu}_{4}$ cases, while they studied those as particular cases of isolated molecules.

On the other hand, for $\mathrm{Cs}_{2} \mathrm{SnAu}_{6}$ and $\mathrm{Cs}_{2} \mathrm{PbAu}_{6}$ the integrated valence electron densities within the atoms obtained with Bader's analysis from DFT and DFT-SOC functionals are shown in Table III and Table IV. In the non-relativistic cases, the sum of all extra electrons in the six $\mathrm{Au}$ atoms is 2.154 and 1.882 , respectively, while Cs and M metal ions together lose 2.158 and 1.887 electrons, respectively. DFT-SOC functionals lead to obtaining 2.154 and 1.882 extra electrons for the six $\mathrm{Au}$ ions, while the lost electrons from Cs and M are 2.150 and 1.854 .

In these systems, the gold suboctahedral (o) structure takes relevance as the $\mathrm{Au}-\mathrm{Au}$ interatomic distances, shown in Table I, remain almost constant, suggesting that interactions of the suboctahedron gold anions shown in Fig. 1 (b) have bonding states that restore the distance between them. This can be seen in Fig. 6 and in Table $\mathrm{V}$, which indicates that in most of the compounds, the Hellman-Feynman forces point inward of the gold suboctahedra. However, in the case of the compounds $\mathrm{Rb}_{2} \mathrm{ZrAu}_{6}$ and $\mathrm{Rb}_{2} \mathrm{HfAu}_{6}$ this is not the case.
TABLE III. Calculated charge per atom through Bader's AIM topological analysis for $\mathrm{Cs}_{2} \mathrm{SnAu}_{6} . e_{i}, \rho_{B}, \rho_{B-S O C}$ and $\Omega_{\mathrm{B}}$ are the same as defined in Table II.

\begin{tabular}{ccccc}
\hline \hline Atom & $e_{i}$ & $\rho_{B}$ & $\rho_{B-S O C}$ & $\Omega_{\mathrm{B}}$ \\
\hline $\mathrm{Au}(1)$ & 11 & 11.340 & 11.338 & 25.72 \\
$\mathrm{Au}(2)$ & 11 & 11.343 & 11.338 & 25.74 \\
$\mathrm{Au} \mathrm{(3)}$ & 11 & 11.358 & 11.359 & 25.86 \\
$\mathrm{Au} \mathrm{(4)}$ & 11 & 11.361 & 11.359 & 25.88 \\
$\mathrm{Au} \mathrm{(5)}$ & 11 & 11.379 & 11.375 & 26.03 \\
$\mathrm{Au}(6)$ & 11 & 11.373 & 11.375 & 25.98 \\
\hline $\mathrm{Cs}(1)$ & 9 & 8.279 & 8.278 & 28.37 \\
$\mathrm{Cs}(2)$ & 9 & 8.279 & 8.278 & 28.37 \\
\hline $\mathrm{Sn}(1)$ & 14 & 13.284 & 13.294 & 20.49 \\
\hline \hline
\end{tabular}

TABLE IV. Calculated charge per atom through Bader's AIM topological analysis for $\mathrm{Cs}_{2} \mathrm{PbAu}_{6} . e_{i}, \rho_{B}, \rho_{B-S O C}$ and $\Omega_{\mathrm{B}}$ are the same as defined in Table II.

\begin{tabular}{ccccc}
\hline \hline Atom & $e_{i}$ & $\rho_{B}$ & $\rho_{B-S O C}$ & $\Omega_{\mathrm{B}}$ \\
\hline $\mathrm{Au}(1)$ & 11 & 11.311 & 11.325 & 26.30 \\
$\mathrm{Au}(2)$ & 11 & 11.314 & 11.325 & 26.32 \\
$\mathrm{Au} \mathrm{(3)}$ & 11 & 11.329 & 11.304 & 26.45 \\
$\mathrm{Au} \mathrm{(4)}$ & 11 & 11.332 & 11.304 & 26.47 \\
$\mathrm{Au} \mathrm{(5)}$ & 11 & 11.301 & 11.296 & 26.23 \\
$\mathrm{Au}(6)$ & 11 & 11.295 & 11.296 & 26.18 \\
\hline $\mathrm{Cs}(1)$ & 9 & 8.274 & 8.274 & 29.50 \\
$\mathrm{Cs}(2)$ & 9 & 8.274 & 8.274 & 29.50 \\
\hline $\mathrm{Pb}(1)$ & 14 & 13.565 & 13.598 & 24.60 \\
\hline \hline
\end{tabular}

The vector forces point outward with a modulus of 1.058 and $0.33710^{-6} \mathrm{eV} / \AA$ per atom, respectively. A consequence of these forces pointing outward the suboctahedra could turn into overlappings in the frequency of the tree-peak ordering caused by gold anions with the alkaliion crests in these compounds, see VDOS of $\mathrm{Rb}_{2} \mathrm{ZrAu}_{6}$ and $\mathrm{Rb}_{2} \mathrm{HfAu}_{6}$ shown in Fig. 4. This phenomenon involves more vibrational interactions between gold anions and the alkali metals than their other cousins.

TABLE V. Modulus of the Hellman-Feynman forces for the dynamically stable compounds shown in Fig. 6 (in $10^{-6}$ $\mathrm{eV} / \AA)$. ( - ) or $(+)$ stands for vector directions inward or outward the gold suboctahedra, respectively.

\begin{tabular}{ccc}
\hline \hline & Direction & Vector Modulus \\
\hline $\mathrm{Rb}_{2} \mathrm{TiAu}_{6}$ & $(-)$ & 1.153 \\
$\mathrm{Rb}_{2} \mathrm{ZrAu}_{6}$ & $(+)$ & 1.058 \\
$\mathrm{Rb}_{2} \mathrm{HfAu}_{6}$ & $(+)$ & 0.337 \\
\hline $\mathrm{K}_{2} \mathrm{TiAu}_{6}$ & $(-)$ & 4.706 \\
$\mathrm{~K}_{2} \mathrm{ZrAu}_{6}$ & $(-)$ & 5.431 \\
$\mathrm{~K}_{2} \mathrm{HfAu}_{6}$ & $(-)$ & 4.331 \\
\hline $\mathrm{Cs}_{2} \mathrm{SnAu}_{6}$ & $(-)$ & 3.763 \\
$\mathrm{Cs}_{2} \mathrm{PbAu}_{6}$ & $(-)$ & 4.961 \\
\hline \hline
\end{tabular}




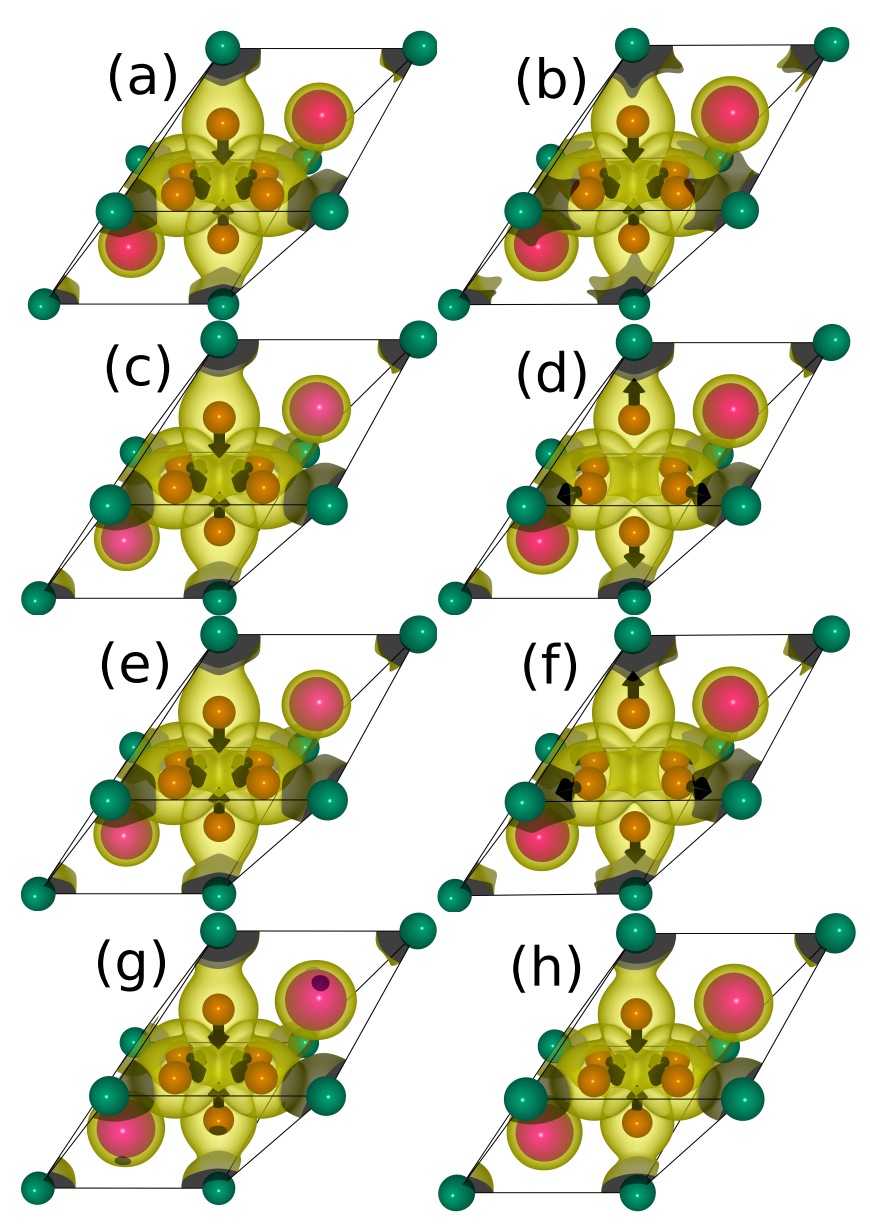

FIG. 6. Primitive cells of the compounds (a) $\mathrm{K}_{2} \mathrm{TiAu}_{6}$, (b) $\mathrm{Rb}_{2} \mathrm{TiAu}_{6}$, (c) $\mathrm{K}_{2} \mathrm{ZrAu}_{6}$, (d) $\mathrm{Rb}_{2} \mathrm{ZrAu}_{6}$, (e) $\mathrm{K}_{2} \mathrm{HfAu}_{6}$, (f) $\mathrm{Rb}_{2} \mathrm{HfAu}_{6}$, (g) $\mathrm{Cs}_{2} \mathrm{SnAu}_{6}$ and (h) $\mathrm{Cs}_{2} \mathrm{PbAu}_{6}$ indicating with a yellow surface the electronic density and with black arrows the overall Hellman-Feynman forces obtained from DFT calculations. The color code for ions is the same as Fig. 1.

\section{ELECTRONIC DENSITY OF STATES AND CRYSTAL ORBITAL HAMILTON POPULATION}

The electronic density of states (EDOS) of the $\mathrm{Rb}_{2} \mathrm{TiAu}_{6}$ compound, shown in Fig. 7 (a), reveals that most of the d-orbital electronic states from $\mathrm{Au}$ anions extend from -5 to $-2 \mathrm{eV}$. Additionally, the pCOHP and IpCOHP analysis depict the bonding states from nearestneighbor interactions from $\mathrm{Ti}-\mathrm{Au}$ (6 interactions) and $\mathrm{Au}-\mathrm{Au}$ (12 interactions). The negative IpCOHP value averaged from the $\mathrm{Ti}-\mathrm{Au}$ interactions at the Fermi level $\left(E_{F}\right)$ is $0.38 \mathrm{eV} /$ pair, indicating the existence of bonds exhibiting some covalent characteristics. On the other hand, the $\mathrm{Au}-\mathrm{Au}$ interactions show that bonding states are present from -5.7 up to $-3.3 \mathrm{eV}$ while antibonding states from -3.3 to $-1.85 \mathrm{eV}$. The negative IpCOHP averaged from all the $\mathrm{Au}-\mathrm{Au}$ interactions approach to zero, indicating nonbonding between gold anions up to the $E_{F}$.

Looking closer at the non-relativistic spin-polarized projected EDOS of the $\mathrm{Rb}_{2} \mathrm{TiAu}_{6}$ compound, shown in

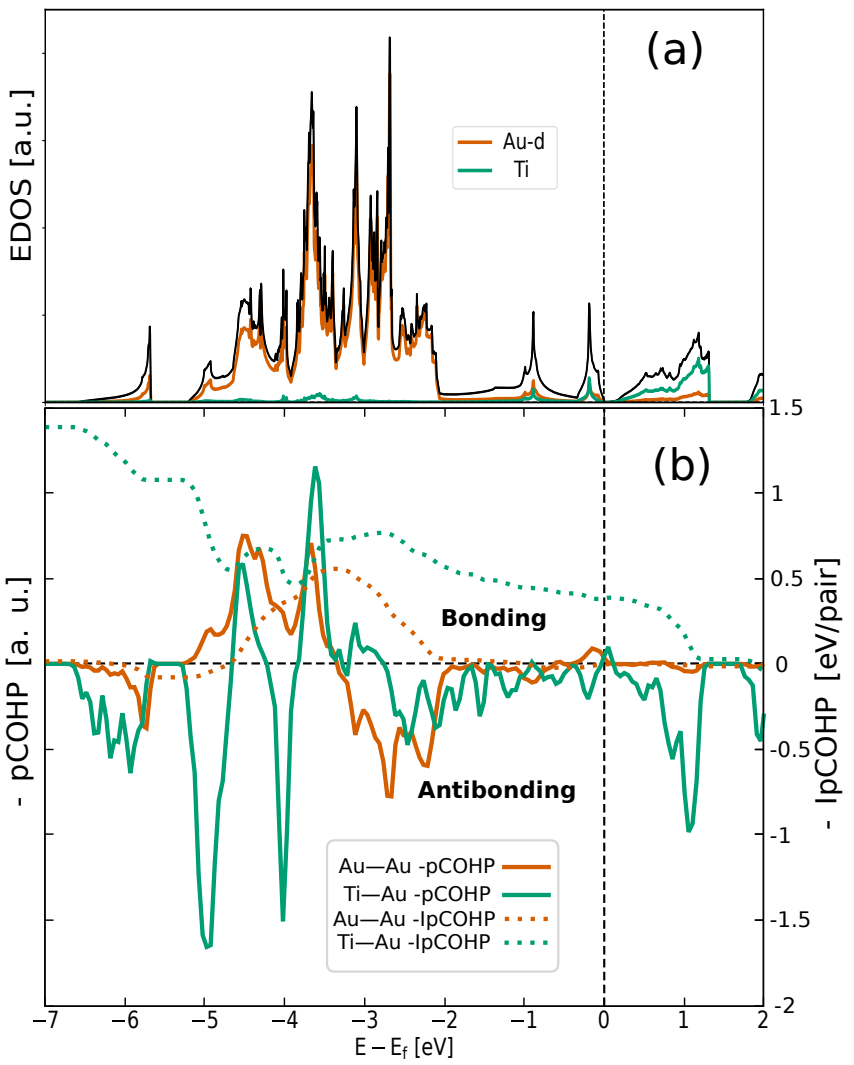

FIG. 7. (a) EDOS with d-orbital contributions from $\mathrm{Au}$ anions and Ti ionic contributions and (b) pCOHP from $\mathrm{Au}-\mathrm{Au}$ and $\mathrm{Ti}-\mathrm{Au}$ interactions of $\mathrm{Rb}_{2} \mathrm{TiAu}_{6}$. The solid black line represents the total EDOS, the dashed vertical black line represents the Fermi level $\left(E_{F}\right)$.

Fig. 8, it is possible to see that the material have equal EDOS values for spin-up and spin-down, manifested in spin isotropic, non magnetic materials. One also can perceive with more detail the orbital contributions from $\mathrm{Au}$ and Ti ions. For example, Fig. 8 (a) reveals that the $5 \mathrm{~d}$ electronic states from Au govern most of the EDOS from -5 to $-2 \mathrm{eV}$ and appear at higher energy levels beyond the $E_{F}$, as previously seen in Fig. 7 (a). On the other hand, 6s electronic states from $\mathrm{Au}$ anions have significant contributions at lower energies, from $-7 \mathrm{eV}$ to -5.7 $\mathrm{eV}$. That low energy peak is caused by a well-separated electronic band that will be discussed in section $\mathrm{D}$. These electronic states also contribute along all the EDOS up to the $E_{F}$ and does so significantly to the highest occupied electronic states, just below the $E_{F}$.

Finally, the $6 \mathrm{p}$ electron states from $\mathrm{Au}$ anions have a broad contribution above the $E_{F}$, indicating that the $6 \mathrm{~s}$ and $5 \mathrm{~d}$ orbitals from $\mathrm{Au}$ are nearly filled owing to its anionic character. That means that this system belongs to a limit of electronic localization. The presence of $6 \mathrm{p}$ electrons above the $E_{F}$ has been previously observed in the conduction bands of alkali auride $\mathrm{AAu}(\mathrm{A}=\mathrm{K}, \mathrm{Rb}$, Cs) compounds with a bandgap ${ }^{[7]}$. However, the material studied here is a poor conductor with conduction 

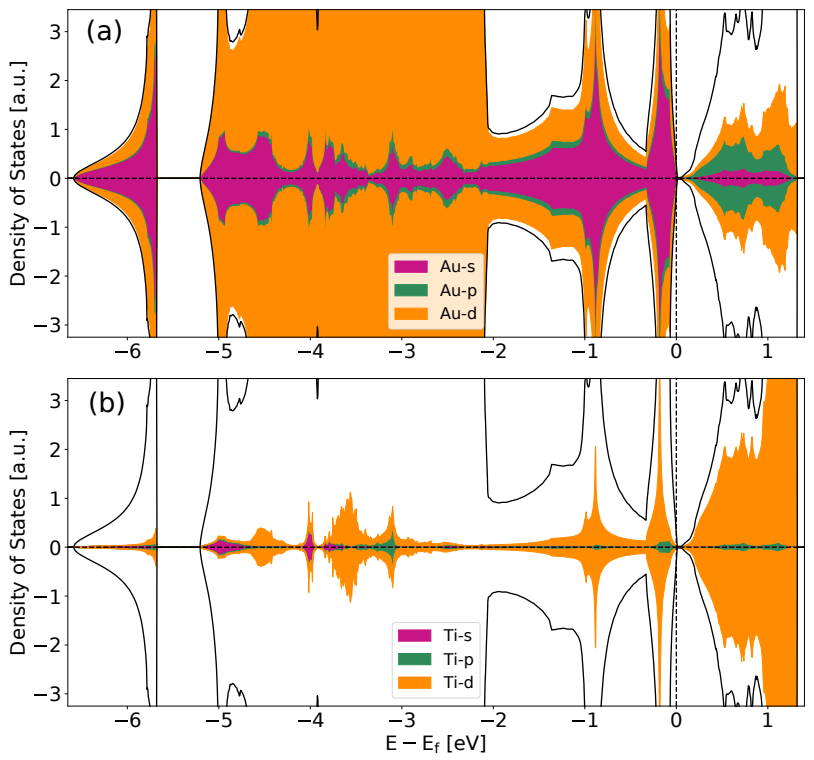

FIG. 8. Projected EDOS from (a) Au ions and (b) Ti ions showing the orbital contributions of $\mathrm{Rb}_{2} \mathrm{TiAu}_{6}$. Positive and negative values of the EDOS indicate spin up and spin down respectively. The solid black line represents the total EDOS, the dashed vertical black line represents the $E_{F}$.

states only around the $\Gamma$ point, as seen in section D. On the other hand, the orbital projections of the Ti metal indicate that most of the conduction states above the $E_{F}$ are formed by d-orbitals.

In contrast, the EDOS, pCOHP and IpCOHP of the $\mathrm{Cs}_{2} \mathrm{SnAu}_{6}$ and $\mathrm{Cs}_{2} \mathrm{PbAu}_{6}$ compounds are shown in Figs. 9-12. Similar to $\mathrm{Rb}_{2} \mathrm{TiAu}_{6}$, the d-orbital electronic states from $\mathrm{Au}$ anions predominate below $-2 \mathrm{eV}$. The pCOHP and IpCOHP analysis show that both $\mathrm{M}-\mathrm{Au}$ and $\mathrm{Au}-\mathrm{Au}$ covalent interactions exist in the two cases. In the case of $\mathrm{Cs}_{2} \mathrm{SnAu}_{6}$, the negative IpCOHP for $\mathrm{Sn}-\mathrm{Au}$ and $\mathrm{Au}-\mathrm{Au}$ interactions are 1.25 and 0.33 $\mathrm{eV} /$ pair. For the $\mathrm{Cs}_{2} \mathrm{PbAu}_{6}$, the negative IpCOHP for $\mathrm{Pb}-\mathrm{Au}$ and $\mathrm{Au}-\mathrm{Au}$ interactions are 1.13 and 0.36 $\mathrm{eV} /$ pair. In both cases, the $\mathrm{M}-\mathrm{Au}$ bonds exhibit more covalent character than the $\mathrm{Au}-\mathrm{Au}$ bonds. The $\mathrm{M}-\mathrm{Au}$ bonding due to the s-orbital electrons in the $\mathrm{Pb}$ compound occurs at lower energy than the one with Sn, being the peaks at -9.1 and $-7.6 \mathrm{eV}$, respectively. The projected EDOS of $\mathrm{Cs}_{2} \mathrm{SnAu}_{6}$ and $\mathrm{Cs}_{2} \mathrm{PbAu}_{6}$ compounds shown in Fig. 11 and 12 are similar near $E_{F}$, sharing an electronic forbidden zone going from -0.63 to $-0.61 \mathrm{eV}$ and -0.78 to $0.64 \mathrm{eV}$, respectively. The s-orbital electronic states from $\mathrm{Au}$ anions are similar to those of the $\mathrm{Rb}_{2} \mathrm{TiAu}_{6}$ compound; however, in these two materials, the 6 s electrons continue to conduction states above the $E_{F}$, and the $6 \mathrm{p}$ electronic states appear hybridized with the major contribution around $+1.5 \mathrm{eV}$. On the other hand, p-orbital electronic states from $\mathrm{Sn}$ and $\mathrm{Pb}$ have contributions below the forbidden zone as well as few conduction states above the $E_{F}$.

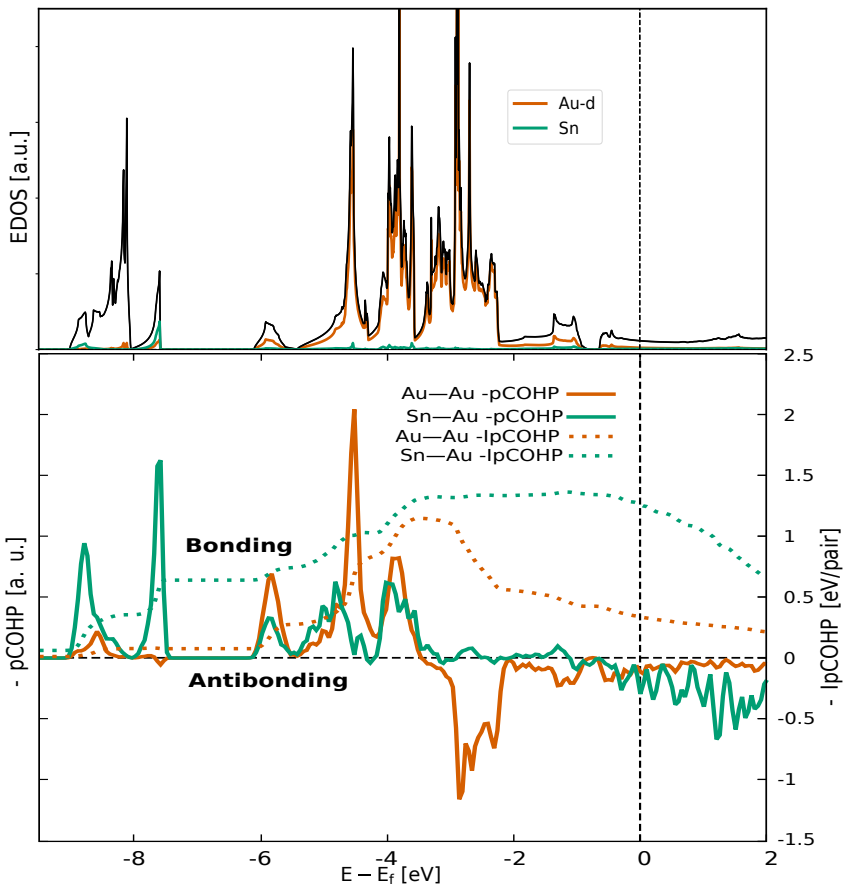

FIG. 9. Top: EDOS with d-orbital contributions from Au anions and Sn ionic contributions and down: COHP from $\mathrm{Au}-\mathrm{Au}$ and $\mathrm{Sn}-\mathrm{Au}$ interactions of $\mathrm{Cs}_{2} \mathrm{SnAu}_{6}$. The solid black line represents the total EDOS, the dashed vertical black line represents the $E_{F}$.

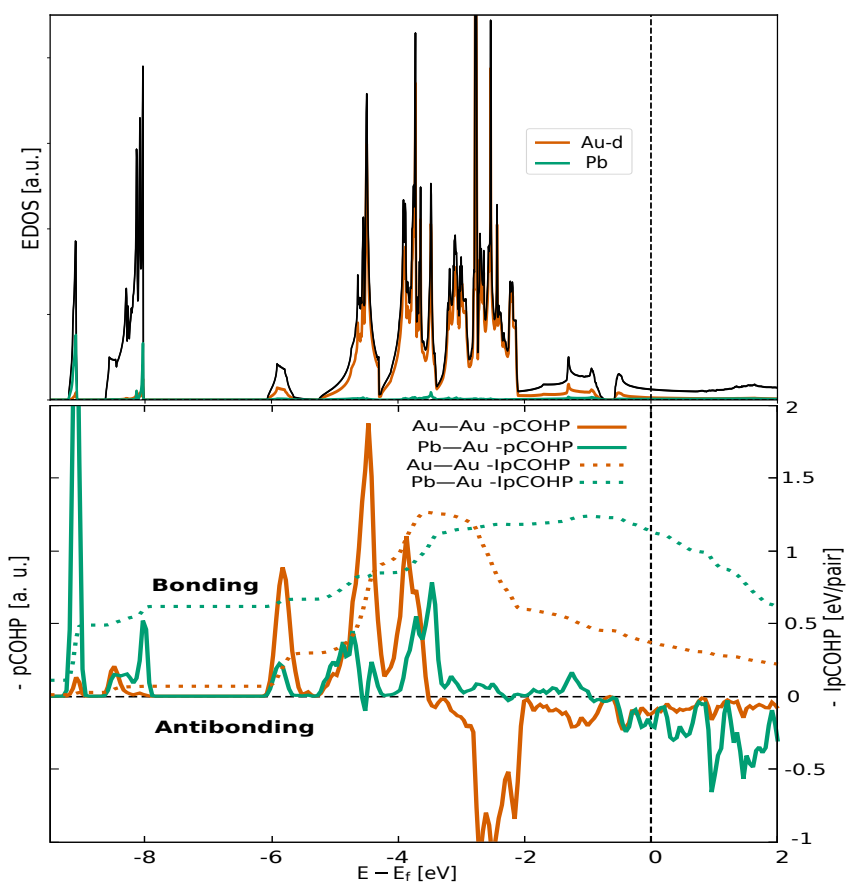

FIG. 10. Top: EDOS with d-orbital contributions from Au anions and $\mathrm{Pb}$ ionic contributions and down: COHP from $\mathrm{Au}-\mathrm{Au}$ and $\mathrm{Pb}-\mathrm{Au}$ interactions of $\mathrm{Cs}_{2} \mathrm{PbAu}_{6}$. The solid black line represents the total EDOS, the dashed vertical black line represents the $E_{F}$. 

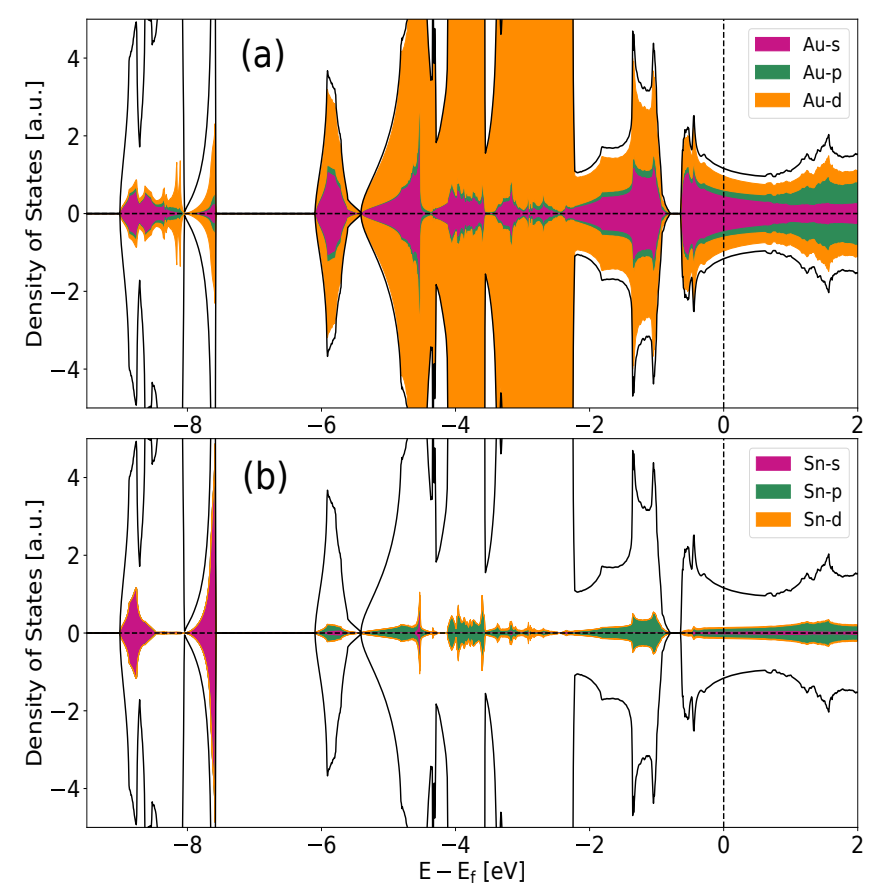

FIG. 11. Projected EDOS from (a) Au ions and (b) Sn ions showing the orbital contributions of $\mathrm{Cs}_{2} \mathrm{SnAu}_{6}$. Positive and negative values of the EDOS indicate spin up and spin down respectively. The solid black line represents the total EDOS, the dashed vertical black line represents the $E_{F}$.
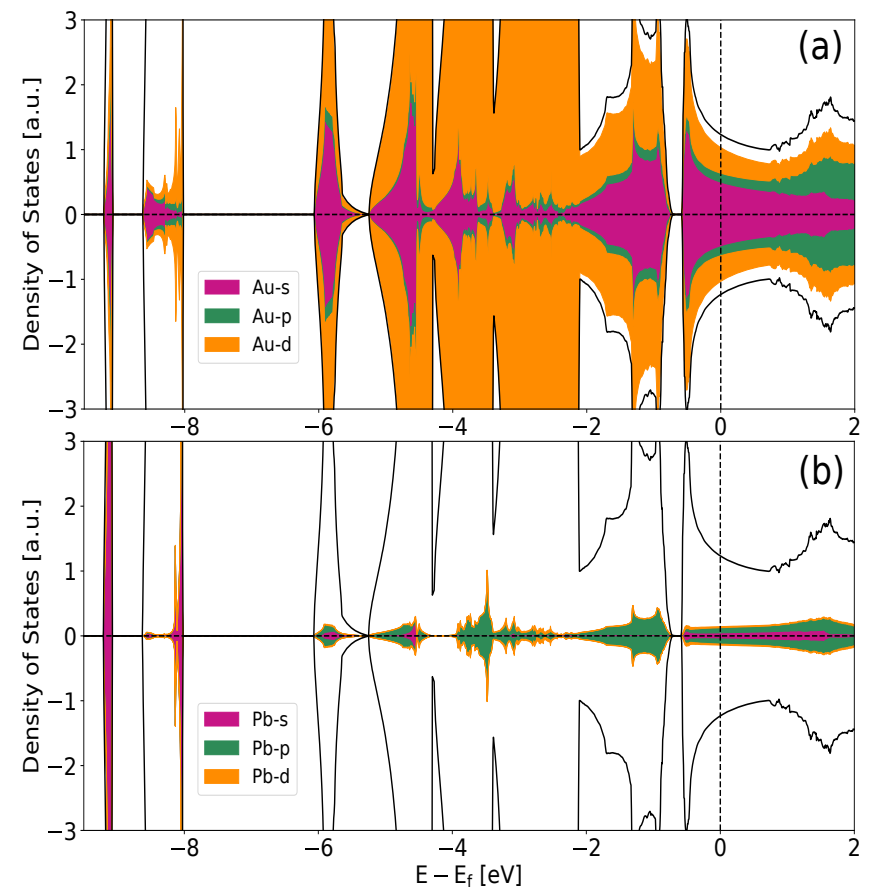

FIG. 12. Projected EDOS from (a) Au ions and (b) Pb ions showing the orbital contributions of $\mathrm{Cs}_{2} \mathrm{PbAu}_{6}$. Positive and negative values of the EDOS indicate spin up and spin down respectively. The solid black line represents the total EDOS, the dashed vertical black line represents the $E_{F}$.
In this auride family gold behaves as an anion with interactions between $\mathrm{M}$ metal atoms with $6 \mathrm{Au}$ anions in the octahedral $(\mathrm{O})$ structure and between $\mathrm{Au}$ atoms in the suboctahedral (o) structure. The remaining pCOHPs and EDOS are shown Figs. 9-13 in the SI, and the negative IpCOHPs for the other 5 compounds are shown in Table VI.

TABLE VI. Negative IpCOHP for the dynamically stable compounds (in $\mathrm{eV} /$ pair). $\mathrm{M}-\mathrm{Au}$ are taken from 6 interactions and $\mathrm{Au}-\mathrm{Au}$ from 12 interactions.

\begin{tabular}{ccc}
\hline \hline & $\mathrm{M}-\mathrm{Au}$ & $\mathrm{Au}-\mathrm{Au}$ \\
\hline $\mathrm{Rb}_{2} \mathrm{TiAu}_{6}$ & 0.38 & 0 \\
$\mathrm{Rb}_{2} \mathrm{ZrAu}_{6}$ & 1.84 & 0.21 \\
$\mathrm{Rb}_{2} \mathrm{HfAu}_{6}$ & 1.66 & 0.19 \\
\hline $\mathrm{K}_{2} \mathrm{TiAu}_{6}$ & 0.35 & -0.02 \\
$\mathrm{~K}_{2} \mathrm{ZrAu}_{6}$ & 1.89 & 0.24 \\
$\mathrm{~K}_{2} \mathrm{HfAu}_{6}$ & 1.68 & 0.22 \\
\hline $\mathrm{Cs}_{2} \mathrm{SnAu}_{6}$ & 1.25 & 0.33 \\
$\mathrm{Cs}_{2} \mathrm{PbAu}_{6}$ & 1.13 & 0.36 \\
\hline \hline
\end{tabular}

\section{ELECTRONIC BAND STRUCTURE}

The calculated electronic band structure of $\mathrm{Rb}_{2} \mathrm{TiAu}_{6}$ with and without SOC is shown in Fig. 13. The SOC calculations unveil some bands with energy degeneracies (in the non-SOC case) on the high symmetry points. For example, around $-0.25 \mathrm{eV}$ at the $\Gamma$ point or near Fermi energy and $-1 \mathrm{eV}$ at the $\mathrm{L}$ point. There are only electronic states at the Fermi energy around the $\Gamma$ point and zero conduction states at the L point, even though electronic mobilities are high.

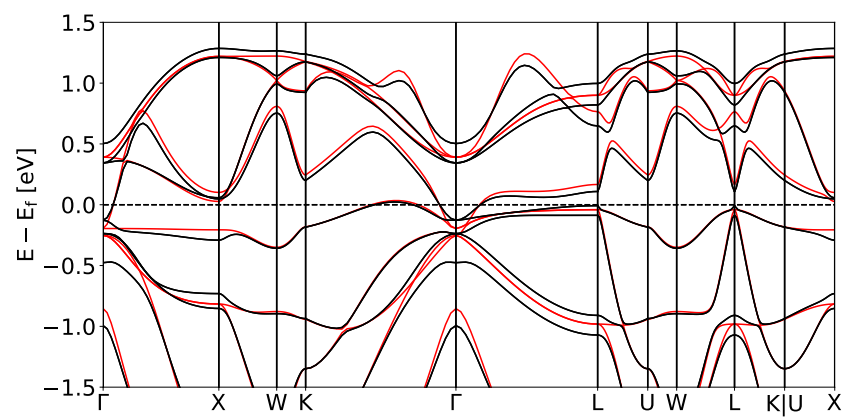

FIG. 13. Computed electronic band structure of $\mathrm{Rb}_{2} \mathrm{TiAu}_{6}$. Black solid lines (red solid lines) represent the band structure with (without) spin-orbit coupling. The dashed line represents the $E_{F}$.

Atomic orbital projections on the band structure, shown in Fig. 14, shows that the 3d orbital states from $\mathrm{Ti}$ atoms have major participation in the conduction states, above the $E_{F}$. The 5 d states from $\mathrm{Au}$ atoms appear mostly from -5 to -2 as previously analyzed in section C. 

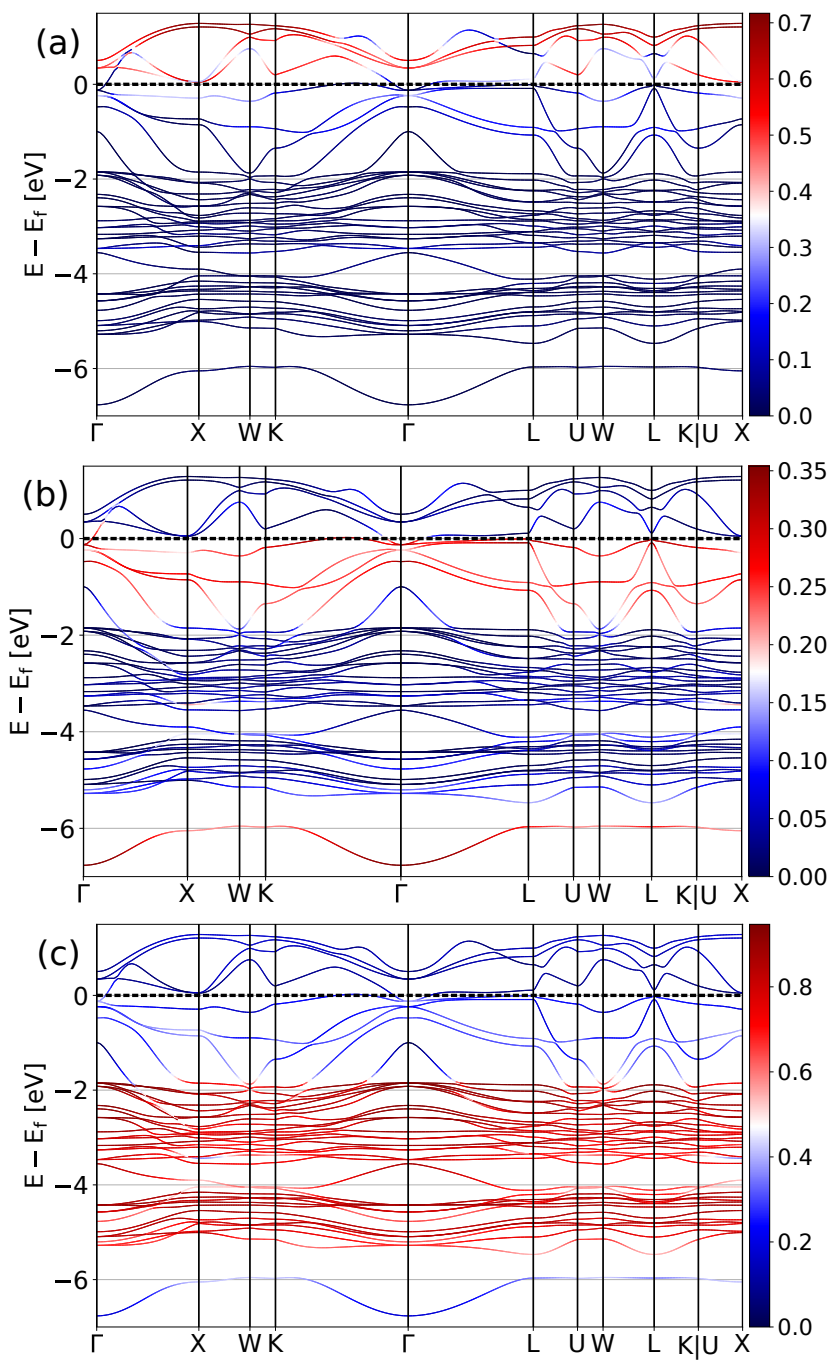

FIG. 14. Projected electronic band structure of $\mathrm{Rb}_{2} \mathrm{TiAu}_{6}$. The red color intensity corresponds to the degree of contribution of (a) $3 d$-orbital from Ti, and (b) $6 s$-orbitals and (c) $5 d$-orbitals from $\mathrm{Au}$. The horizontal dashed line represents the Fermi level.

Lastly, the 6s electronic states from Au atoms have major participation near the conduction states, just below the $E_{F}$; however, there is also a band from -7 to $-5.7 \mathrm{eV}$ that form the low energy peak mentioned in section C. A very similar band has been previously observed in the isostructural compounds studied by Halilov et al. (ref. [47]). However, the band from that work is caused by s-states from hydrogen atoms, which again opens the question if gold behaves as a halogen or as hydrogen $[21,48,49]$.

On the other hand, the calculated electronic band structure of $\mathrm{Cs}_{2} \mathrm{SnAu}_{6}$ and $\mathrm{Cs}_{2} \mathrm{PbAu}_{6}$ near the $E_{F}$ are shown in Fig. 15. Like the previous case, in the absence of SOC, there are electronic bands with energy degeneracies on the high symmetry points as $\mathrm{L}, \Gamma$, and $\mathrm{X}$, that are unfolded by the calculations considering SOC. However, in contrast to the compounds with group 4 tetravalent metals, in these two compounds there are electronic
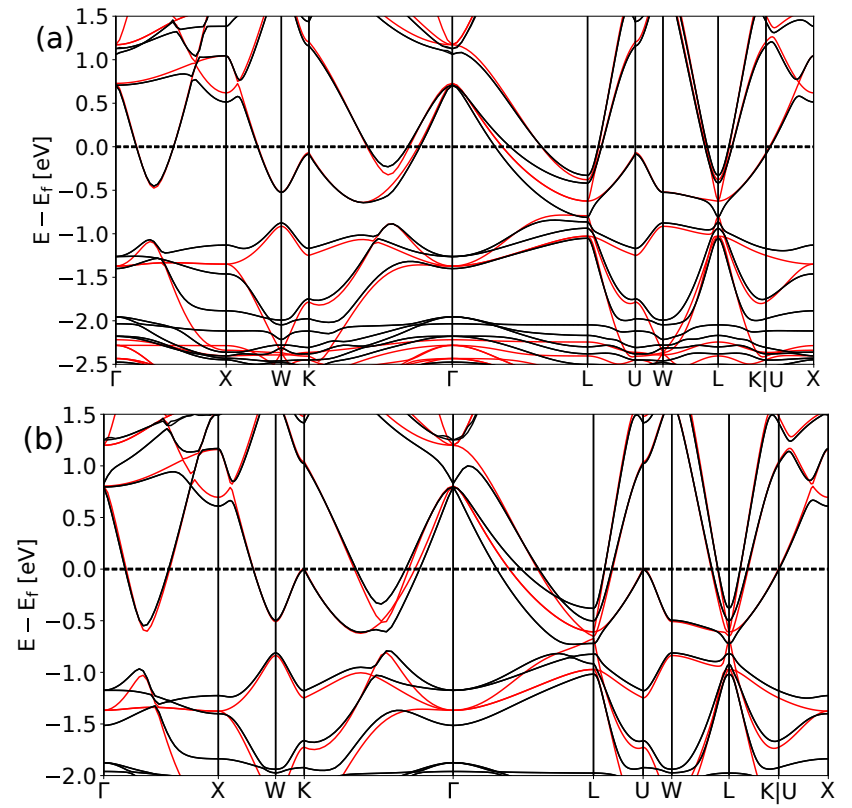

FIG. 15. Computed electronic band structure of $\mathrm{Cs}_{2} \mathrm{SnAu}_{6}$ and $\mathrm{Cs}_{2} \mathrm{PbAu}_{6}$. Black solid lines (red solid lines) represent the band structure with (without) spin-orbit coupling. The dashed line represents the $E_{F}$.

states over the $E_{F}$ around almost all the Brillouin zone, including an electron pocket in the $\Gamma-\mathrm{X}$ momentum path, which indicates a more metallic behavior. One can also see the forbidden energetic zones mentioned in section $\mathrm{C}$. The difference between the electronic structure of the previous case and these two is due to the type of the valence electron orbitals from the $\mathrm{M}$ tetravalent metal, in which the ones of group 4 possess $d$ type orbitals, while the ones of group 14 possess $p$ type orbitals.

In Fig. 16 the projected atomic orbitals of the $\mathrm{Cs}_{2} \mathrm{SnAu}_{6}$ compound are shown, representing also the general chemistry of the $\mathrm{Cs}_{2} \mathrm{PbAu}_{6}$ compound. For example, in Fig. 16 (a) the $5 p$ orbitals from Sn are shown, which are in occupied and unoccupied states that go from -4 to $-3 \mathrm{eV}$ at the $\mathrm{X}$ and $\mathrm{W}$ points, up to energetic levels beyond the $E_{F}$ around the $\Gamma$ point. Conversely, the $5 \mathrm{~d}$ and $6 \mathrm{~s}$ states from $\mathrm{Au}$ anions present similar characteristics to the previous case observed in Fig. 14 for the $\mathrm{Rb}_{2} \mathrm{TiAu}_{6}$ compound, in which the contributions from the $5 \mathrm{~d}$ states are below $-2 \mathrm{eV}$ and the ones from $6 \mathrm{~s}$ states are near the $E_{F}$. In these two compounds, the $6 \mathrm{~s}$ states from $\mathrm{Au}$ anions are in the last occupied states; however, there are also unoccupied electronic bands with significant contributions of $6 \mathbf{s}$ states, as shown in Fig. 16 (b).

The remaining electronic band structures of the auride subfamily with group 4 tetravalent metals are shown in Figs. $14-18$ of the SI. 

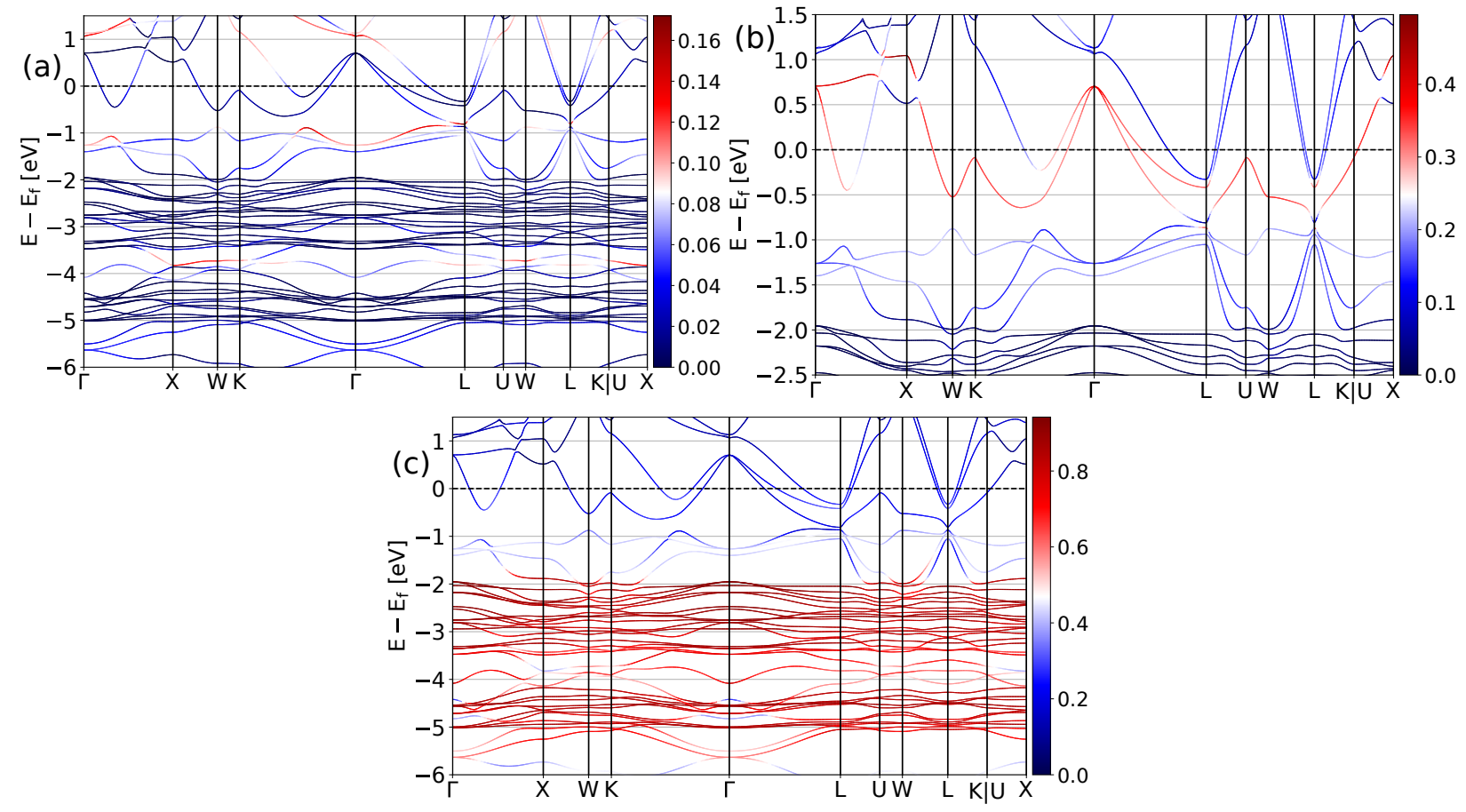

FIG. 16. Projected electronic band structure of $\mathrm{Cs}_{2} \mathrm{SnAu}_{6}$. The red color intensity corresponds to the degree of contribution of (a) $5 p$-orbital from $\mathrm{Sn}$, and (b) $6 s$-orbitals and (c) $5 d$-orbitals from Au. The horizontal dashed line represents the Fermi level.

\section{CONCLUSIONS AND OUTLOOK}

First-principles phonon-structure calculations demonstrated that the eight compounds $\mathrm{Rb}_{2} \mathrm{TiAu}_{6}, \mathrm{Rb}_{2} \mathrm{ZrAu}_{6}$, $\mathrm{Rb}_{2} \mathrm{HfAu}_{6}, \mathrm{~K}_{2} \mathrm{TiAu}_{6}, \mathrm{~K}_{2} \mathrm{ZrAu}_{6}, \mathrm{~K}_{2} \mathrm{HfAu}_{6}, \mathrm{Cs}_{2} \mathrm{PbAu}_{6}$ and $\mathrm{Cs}_{2} \mathrm{SnAu}_{6}$ are dynamically stable with the double perovskite structure within the cubic $F m \overline{3} m$ space group $\left(\mathrm{K}_{2} \mathrm{PtCl}_{6}\right.$-type). The phonon gap variations in these materials could lead to future phonon structure engineering. Bader AIM topological analysis shows an anionic character in $\mathrm{Au}$ atoms. However, more complex $\mathrm{Au}-\mathrm{M}$ and $\mathrm{Au}-\mathrm{Au}$ covalent interactions were analyzed by the Crystal Orbital Hamilton Population (COHP) method. Finally, electronic band structure calculations show energy splittings generally caused by heavy atoms with strong spin-orbit interactions. In the subfamily with group 4 M metals, the conduction states near the Fermi energy are governed by 6 s states from $\mathrm{Au}$ atoms surrounding the zero-momentum $\Gamma$ point. Conversely, in the subfamily with group $14 \mathrm{M}$ metals, the last occupied electronic states near the Fermi energy are also governed by $6 \mathrm{~s}$ states from gold. However, there exist unoccupied 6 s electronic states and states over the Fermi level along all the first Brillouin zone and an electron pocket in the $\Gamma-\mathrm{X}$ momentum path. The differences in the band structures between both subfamilies are attributed to the interaction of the $d$ or $p$ electronic states from the M ion with the six $\mathrm{Au}$ anions in the octahedral geometry.
[1] G. Kienast, J. Verma, and W. Klemm, Das verhalten der alkalimetalle zu kupfer, silber und gold, Zeitschrift für anorganische und allgemeine Chemie 310, 143.

[2] W. E. Spicer, A. H. Sommer, and J. G. White, Studies of the semiconducting properties of the compound $\mathrm{CsAu}$, Phys. Rev. 115, 57 (1959).

[3] A. D. Pelton, The AuCs (Gold-Cesium) system, Bulletin of Alloy Phase Diagrams 7, 19 (1986).

[4] A. D. Pelton, The AuRb (Gold-Rubidium) system, Bulletin of Alloy Phase Diagrams 7, 139 (1986).

[5] G. Tinelli and D. Holcomb, NMR and structural properties of CsAu and RbAu, Journal of Solid State Chemistry 25, 157 (1978).
[6] T. L. Liu, Optical properties of RbAu and CsAu, Phys. Rev. B 12, 3008 (1975).

[7] M. Miao, J. Brgoch, A. Krishnapriyan, A. Goldman, J. A. Kurzman, and R. Seshadri, On the stereochemical inertness of the auride lone pair: Ab initio studies of AAu (A $=\mathrm{K}, \mathrm{Rb}, \mathrm{Cs}$ ), Inorganic Chemistry 52, 8183 (2013).

[8] C. Feldmann and M. Jansen, $\mathrm{Cs}_{3} \mathrm{AuO}$, the first ternary oxide with anionic gold, Angewandte Chemie International Edition in English 32, 1049 (1993), https://onlinelibrary.wiley.com/doi/pdf/10.1002/anie.199310491.

[9] A. Pantelouris, G. Kueper, J. Hormes, C. Feldmann, and M. Jansen, Anionic gold in $\mathrm{Cs}_{3} \mathrm{AuO}$ and $\mathrm{Rb}_{3} \mathrm{AuO}$ established by X-ray absorption spectroscopy, Journal of the American Chemical Society 117, 11749 (1995). 
[10] A. Karpov, J. Nuss, U. Wedig, and M. Jansen, Covalently bonded [Pt]- chains in bapt: extension of the zintlklemm concept to anionic transition metals?, Journal of the American Chemical Society 126, 14123 (2004).

[11] A. Karpov, M. Konuma, and M. Jansen, An experimental proof for negative oxidation states of platinum: ESCAmeasurements on barium platinides, Chem. Commun. , 838 (2006).

[12] T. Andersen, H. K. Haugen, and H. Hotop, Binding energies in atomic negative ions: III, Journal of Physical and Chemical Reference Data 28, 1511 (1999), https://doi.org/10.1063/1.556047.

[13] A.-V. Mudring, M. Jansen, J. Daniels, S. Krämer, M. Mehring, J. P. Prates Ramalho, A. H. Romero, and M. Parrinello, Cesiumauride Ammonia (1/1), $\mathrm{CsAu} \cdot \mathrm{NH}_{3}$ : A crystalline analogue to alkali metals dissolved in ammonia?, Angewandte Chemie International Edition 41, 120.

[14] P. D. C. Dietzel and M. Jansen, Synthesis and crystal structure determination of tetramethylammonium auride, Chem. Commun., 2208 (2001).

[15] M. Jansen, The chemistry of gold as an anion, Chem. Soc. Rev. 37, 1826 (2008).

[16] M. Jansen, Effects of relativistic motion of electrons on the chemistry of gold and platinum, Solid State Sciences 7, 1464 (2005), special issue in honour of C.N.R. Rao.

[17] P. Pyykko and J. P. Desclaux, Relativity and the periodic system of elements, Accounts of Chemical Research 12, 276 (1979), https://doi.org/10.1021/ar50140a002.

[18] P. Schwerdtfeger, P. D. W. Boyd, A. K. Burrell, W. T. Robinson, and M. J. Taylor, Relativistic effects in gold chemistry. 3. gold(I) complexes, Inorganic Chemistry 29, 3593 (1990).

[19] P. Pyykkö, Relativity, gold, closed-shell interactions, and $\mathrm{CsAu} \cdot \mathrm{NH}_{3}$, Angewandte Chemie International Edition 41, 3573 .

[20] P. Schwerdtfeger, Relativistic effects in properties of gold, Heteroatom Chemistry 13, 578 (2002), https://onlinelibrary.wiley.com/doi/pdf/10.1002/hc.10093

[21] L. Gagliardi, When does gold behave as a halogen? predicted uranium tetraauride and other $\mathrm{MAu}_{4}$ tetrahedral species, (M = Ti, Zr, Hf, Th), Journal of the American Chemical Society 125, 7504 (2003).

[22] Y. Erdogdu, T. Jian, G. V. Lopez, W.-L. Li, and L.-S. Wang, On the electronic structure and chemical bonding of titanium tetraauride: $\mathrm{TiAu}_{4}$ and $\mathrm{TiAu}_{4}^{-}$, Chemical Physics Letters 610-611, 23 (2014).

[23] A. Kojima, K. Teshima, Y. Shirai, and T. Miyasaka, Organometal halide perovskites as visible-light sensitizers for photovoltaic cells, Journal of the American Chemical Society 131, 6050 (2009).

[24] M.-G. Ju, M. Chen, Y. Zhou, H. F. Garces, J. Dai, L. Ma, N. P. Padture, and X. C. Zeng, Earth-abundant nontoxic titanium(IV)-based vacancy-ordered double perovskite halides with tunable 1.0 to 1.8 ev bandgaps for photovoltaic applications, ACS Energy Letters 3, 297 (2018).

[25] W. Kohn and L. J. Sham, Self-consistent equations including exchange and correlation effects, Phys. Rev. 140, A1133 (1965).

[26] Efficiency of ab-initio total energy calculations for metals and semiconductors using a plane-wave basis set, Computational Materials Science 6, 15 (1996).

[27] J. P. Perdew, K. Burke, and M. Ernzerhof, Generalized gradient approximation made simple, Phys. Rev. Lett.
77, 3865 (1996)

[28] P. E. Blöchl, Projector augmented-wave method, Phys. Rev. B 50, 17953 (1994).

[29] S. Steiner, S. Khmelevskyi, M. Marsmann, and G. Kresse, Calculation of the magnetic anisotropy with projected-augmented-wave methodology and the case study of disordered $\mathrm{Fe}_{1-x} \mathrm{Co}_{x}$ alloys, Phys. Rev. B 93, 224425 (2016).

[30] S. Grimme, J. Antony, S. Ehrlich, and H. Krieg, A consistent and accurate ab initio parametrization of density functional dispersion correction (DFT-D) for the 94 elements $\mathrm{H}-\mathrm{Pu}$, The Journal of Chemical Physics 132, 154104 (2010), https://doi.org/10.1063/1.3382344.

[31] H. J. Monkhorst and J. D. Pack, Special points for Brillouin-zone integrations, Phys. Rev. B 13, 5188 (1976).

[32] U. Herath, P. Tavadze, X. He, E. Bousquet, S. Singh, F. Muñoz, and A. H. Romero, Pyprocar: A python library for electronic structure pre/post-processing, Computer Physics Communications 251, 107080 (2020).

[33] S. Curtarolo, W. Setyawan, G. L. Hart, M. Jahnatek, R. V. Chepulskii, R. H. Taylor, S. Wang, J. Xue, K. Yang, O. Levy, M. J. Mehl, H. T. Stokes, D. O. Demchenko, and D. Morgan, Aflow: An automatic framework for highthroughput materials discovery, Computational Materials Science 58, 218 (2012).

[34] R. F. W. Bader, Atoms in Moleculres: A Quantum Theory (Oxford University Press, 1994).

[35] M. Yu and D. R. Trinkle, Accurate and efficient algorithm for bader charge integration, The Journal of Chemical Physics 134, 064111 (2011), https://doi.org/10.1063/1.3553716.

[36] G. Henkelman, A. Arnaldsson, and H. Jónsson, A fast and robust algorithm for bader decomposition of charge density, Computational Materials Science 36, 354 (2006).

[37] E. Sanville, S. D. Kenny, R. Smith, and G. Henkelman, Improved grid-based algorithm for bader charge allocation, Journal of Computational Chemistry 28, 899 (2007), https://onlinelibrary.wiley.com/doi/pdf/10.1002/jcc.20575.

[38] W. Tang, E. Sanville, and G. Henkelman, A grid-based bader analysis algorithm without lattice bias, Journal of Physics: Condensed Matter 21, 084204 (2009).

[39] R. Dronskowski and P. E. Bloechl, Crystal orbital hamilton populations (COHP): energy-resolved visualization of chemical bonding in solids based on density-functional calculations, The Journal of Physical Chemistry 97, 8617 (1993), https://doi.org/10.1021/j100135a014.

[40] S. Maintz, V. L. Deringer, A. L. Tchougréeff, and R. Dronskowski, LOBSTER: A tool to extract chemical bonding from plane-wave based DFT, Journal of Computational Chemistry 37, 1030 (2016), https://onlinelibrary.wiley.com/doi/pdf/10.1002/jcc.24300.

[41] V. L. Deringer, A. L. Tchougréeff, and R. Dronskowski, Crystal orbital hamilton population (COHP) analysis as projected from plane-wave basis sets, The Journal of Physical Chemistry A 115, 5461 (2011).

[42] A. Togo and I. Tanaka, First principles phonon calculations in materials science, Scr. Mater. 108, 1 (2015).

[43] V. Luaña, A. Costales, and A. Martín Pendás, Ions in crystals: The topology of the electron density in ionic materials.II. the cubic alkali halide perovskites, Phys. Rev. B 55, 4285 (1997). 
[44] J.-W. Jiang, Phonon bandgap engineering of strained monolayer $\mathrm{MoS}_{2}$, Nanoscale 6, 8326 (2014).

[45] Y. Yin, B. Wang, Y. E, J. Yao, L. Wang, X. Bai, and W. Liu, Raman spectra and phonon structures of $\mathrm{BaGa}_{4} \mathrm{Se}_{7}$ crystal, Communications Physics 3, 34 (2020).

[46] L. Belpassi, F. Tarantelli, A. Sgamellotti, and H. M. Quiney, The electronic structure of alkali aurides. a fourcomponent dirackohnsham study, The Journal of Physical Chemistry A 110, 4543 (2006).

[47] S. V. Halilov, D. J. Singh, M. Gupta, and R. Gupta, Stability and electronic structure of the complex $\mathrm{K}_{2} \mathrm{PtCl}_{6}$ structure hydrides $\mathrm{DMH}_{6}(\mathrm{D}=\mathrm{Mg}, \mathrm{Ca}, \mathrm{Sr} ; \mathrm{M}=\mathrm{Fe}, \mathrm{Ru}$,
Os), Phys. Rev. B 70, 195117 (2004).

[48] H.-J. Zhai, L.-S. Wang, D. Y. Zubarev, and A. I. Boldyrev, Gold apes hydrogen. the structure and bonding in the planar $\mathrm{B}_{7} \mathrm{Au}_{2}^{-}$and $\mathrm{B}_{7} \mathrm{Au}_{2}$ clusters, The Journal of Physical Chemistry A 110, 1689 (2006).

[49] X. Li, B. Kiran, and L.-S. Wang, Gold as hydrogen. an experimental and theoretical study of the structures and bonding in disilicon gold clusters $\mathrm{Si}_{2} \mathrm{Au}_{n}^{-}$and $\mathrm{Si}_{2} \mathrm{Au}_{n}$ (n $=2$ and 4) and comparisons to $\mathrm{Si}_{2} \mathrm{H}_{2}$ and $\mathrm{Si}_{2} \mathrm{H}_{4}$, The Journal of Physical Chemistry A 109, 4366 (2005). 